Original Research Paper

\title{
Free Vibration Analysis of Rectangular Cantilever Plates Using the Hyperbolic-Trigonometric Series
}

\author{
Mikhail V. Sukhoterin, Sergey O. Baryshnikov and Dmitry A. Aksenov \\ Admiral Makarov State University of Maritime and Inland Shipping, Saint-Petersburg, Russia
}

\author{
Article history \\ Received: 16-08-2016 \\ Revised: 22-12-2016 \\ Accepted: 23-12-2016 \\ Corresponding Author: \\ Mikhail V. Sukhoterin \\ Admiral Makarov State \\ University of Maritime and \\ Inland Shipping, Saint- \\ Petersburg, Russia \\ Email: mv@sukhoterin.com
}

\begin{abstract}
The purpose of this paper is to develop a numerical analytical method for an accurate solution to the problem on frequencies and mode shapes of a rectangular cantilever plate. The problem is reduced to an infinite system of linear algebraic equations relative to ratios of trigonometric series, which contains vibration frequency as a parameter. The core of the method is using two hyperbolic-trigonometric series by two coordinates with six undetermined ratios. Functional series are subject to the main differential equation of vibrations and undetermined ratios are obtained from boundary conditions of the problem. Symmetric and asymmetric mode shapes are considered separately. The symmetric solution required the introduction of an additional function to compensate free terms in the decomposition of hyperbolic functions into Fourier series. The infinite system relative to six successions of undetermined ratios was reduced to a homogeneous infinite system relative to one (basic) succession of ratios. The iterative process of its solution at the chosen vibration frequency was presented. A compact resolving system of homogeneous linear equations was obtained, relative to basic ratios of mode shapes of a rectangular cantilever plate. The search for natural frequencies was done with simple exhaustion of a frequency parameter up to the values, at which the basic ratios become invariable, starting with some iteration. The simplicity of the algorithm and the resolving system allows fast obtaining natural frequencies with high accuracy. The calculation accuracy is analyzed. The results in this study are well coincided with the results of the authors, who fulfilled all the problem's conditions most accurately. The obtained results can be used to do highly accurate dynamic calculations in nanoengineering. The calculation accuracy with this algorithm can be enhanced by increasing the number of terms in series, the number of iterations and the size of the mantissa.
\end{abstract}

Keywords: Accurate Natural Frequency, Rectangular Cantilever Plate

\section{Introduction}

The problem of free vibrations of rectangular cantilever plates does not have an exact closed-form solution. Some authors use methods that allow finding an approximate solution using a simple computational procedure, others tend to satisfy all conditions of the problem more precisely, which causes considerable difficulties of analytical and computational nature. The numerical results of such computations often differ from each other.

A rectangular cantilever plate is a computational scheme for many elements of different constructions, devices and plants. Modern development of aviation and space machinery, nanotechnologies, etc. requires highprecision dynamic computations of these elements.
The goal of this work is to find an accurate solution to the problem, which is an infinite system of linear algebraic equations relative to the ratios of hyperbolictrigonometric function series satisfying all conditions of the problem. Selection of frequencies that give nontrivial solutions of a reduced system and also an increase in the dimension of this system allow obtaining an accurate solution within the limit.

A brief analysis of works on this issue enabled us to concentrate on those which have numerical results for a square plate, which is commonly considered a standard plate. Dividing the major well-known works into theoretical and experimental ones, we put their numerical results in a chronological order in Table 1. 
Table 1. Comparison of the relative natural frequencies of the square cantilever plate $\left(\left(\omega=p b^{2} \sqrt{\rho h / D}\right)\right) v=0.3$

\begin{tabular}{|c|c|c|c|c|c|c|c|c|}
\hline Eigen frequencies & $\omega_{1 \mathrm{~S}}$ & $\omega_{2 \mathrm{~A}}$ & $\omega_{3 \mathrm{~S}}$ & $\omega_{4 \mathrm{~S}}$ & $\omega_{5 \mathrm{~A}}$ & $\omega_{6 \mathrm{~S}}$ & $\omega_{7 \mathrm{~S}}$ & $\omega_{8 \mathrm{~A}}$ \\
\hline Present theory & 3.471 & 8.5061 & 21.284 & 27.1987 & 30.9543 & 54.1837 & - & 64.143 \\
\hline Young (1950) & 3.494 & 8.547 & 21.44 & 27.46 & 31.17 & - & - & - \\
\hline Barton (1951) & 3.494 & 8.547 & 21.44 & 27.46 & 31.17 & - & - & - \\
\hline Claassen and Thorne (1962) & 3.4721 & 8.547 & 21.289 & 27.24 & 31.089 & - & - & - \\
\hline Bazley et al. (1967) & 3.482 & - & 21.365 & 27.276 & - & 54.288 & 61.449 & - \\
\hline Bazley et al. (1967) & 3.43 & - & 20.873 & 26.5 & - & 51.49 & 60.24 & - \\
\hline Anderson et al. (1968) & 3.47 & 8.54 & 21.559 & 27.215 & - & - & - & - \\
\hline Leissa (1973) & 3.4917 & 8.5246 & 21.429 & 27.331 & 31.111 & 54.443 & - & - \\
\hline Gorman (1976) & 3.459 & 8.356 & 21.09 & 27.06 & 30.55 & 53.53 & 61.12 & 63.62 \\
\hline Gorman (1976) & 3.471 & 8.506 & - & - & - & - & 61.25 & - \\
\hline Misusawa (1986) & 3.467 & 8.462 & 21.19 & 27.18 & 30.77 & - & - & - \\
\hline Fu and Price (1987) & 3.473 & 8.516 & 21.42 & 27.15 & 31.03 & - & - & - \\
\hline Liew et al. (1990) & 3.49 & 8.54 & 21.33 & 27.81 & - & - & - & - \\
\hline Bardell (1991) & 3.47 & 8.51 & 21.29 & 27.2 & - & - & - & - \\
\hline Rossi and Laura (1996) & 3.471 & 8.508 & 21.29 & 27.2 & 30.96 & 54.19 & 61.26 & 64.15 \\
\hline Seok et al. (2004) & 3.4603 & 8.4977 & 21.266 & 27.178 & 30.908 & 54.09 & - & - \\
\hline Kerboua et al. (2007) & 3.47 & 8.51 & 21.35 & 27.14 & 30.96 & 53.82 & - & - \\
\hline Wu et al. (2008) & 3.485 & 8.547 & 21.85 & 27 & 31.53 & - & - & - \\
\hline Eftekhari and Jafari (2013) & 3.4306 & 8.0605 & 20.0895 & 25.4991 & 28.2448 & 47.531 & 54.116 & 57.0137 \\
\hline Zhong et al. (2013) & 3.448 & 8.333 & 20.87 & 26.92 & 30.46 & 53.4 & 61.19 & 63.51 \\
\hline $\begin{array}{l}\text { Shi et al. (2014) } \\
\text { experiment }\end{array}$ & 3.435 & 8.063 & 20.085 & 25.493 & 28.247 & 47.532 & 54.066 & 56.987 \\
\hline Barton (1951) & 3.43 & 8.32 & 20.55 & 27.15 & 29.75 & - & - & - \\
\hline Dalley and Ripperger (1952) & 3.37 & 8.26 & 20.55 & 27.15 & 29.75 & - & - & - \\
\hline Lindholm et al. (1965) & 3.38 & 8.46 & 20.74 & 27.45 & 30.43 & - & - & \\
\hline Leissa (1969) & 3.35 & 8.53 & 20.9 & 26.72 & 30.61 & - & - & - \\
\hline Singal et al. (1992) & 3.27 & 7.86 & 20.21 & 26.31 & 29.29 & 51.6 & - & - \\
\hline
\end{tabular}

The first theoretical works on determining natural frequencies and mode shapes of rectangular cantilever plates were published by (Young, 1950; Barton, 1951). They used the Ritz method (Rayleigh-Ritz method), keeping in the approximation expression of the bending function nine items-combinations of hyperbolic and trigonometric functions. The calculated values in (Young, 1950; Barton, 1951) of the first five natural frequencies coincide and serve as the main reference point for many researchers. Many authors (Warburton, 1954; Martin, 1956; Claassen and Thorne, 1962; Leissa, 1973; Mizusawa, 1986; Liew et al., 1990; Wu et al., 2009; Eftekhari and Jafari, 2013; Shi et al., 2014) solved the problem with the same method. Warburton (1954) obtained approximate formulae of frequencies for different boundary conditions, including ones for cantilever plates. A cantilever plate with the ratio of its sides 1.855 was chosen as an example. A similar example was given by Martin (1956). Claassen and Thorne (1962) improved the solutions of Barton (1951) for more exact approximations using computing. He got the first eight natural frequencies of the square cantilever plate's vibration. Leissa (1973) searched for a solution in the form of a double power series with 36 items. Mizusawa (1986) used B-spline functions. He also studied the influence of Poisson's ratio on natural frequencies. Liew et al. (1990) built orthogonal polynomials using the Gram-Schmidt process, which enables speeding up the process of approximation. The first four frequencies were described in the work. Wu et al. (2009) chose the approximation function in the form of double reduced series with combinations of hyperbolic and trigonometric functions $(25 \times 25)$. Eftekhari and Jafari (2013; Shi et al., 2014) analyzed the free vibrations of Mindlin plates using the method of Rayleigh-Ritz.

Bazley et al. (1967) used the Rayleigh-Ritz method for the upper estimate of symmetric vibrations natural frequencies in higher approximations and for the lower estimate they suggest their own variation method, allowing for only a part of the expression of the plate's potential energy.

The Finite Element Method (FEM), initiated, as considered, by Courant (1943), was used by Anderson et al. (1968; Fu and Price, 1987; Bardell, 1991; Rossi and Laura, 1996; Kerboua et al., 2007). Anderson et al. (1968) studied the effect of different gridworks on the solution. The most acceptable numerical solutions are obtained by dividing the plate into 50 triangle elements. Fu and Price (1987) considered vibrations of cantilever plates in air and water. They used a linear hydroelastic theory. The numerical results are obtained for the grid of 16 and 64 elements. Bardell (1991) also applied FEM. The approximation function contains $10 \times 10$ polynomials 
by two coordinates. We use the principle of minimum potential energy of the plate and analyzed the dependency of natural frequencies on Poisson's ratio. Rossi and Laura (1996) used a grid of 100 elements with 121 nodes. Kerboua et al. (2007) applied semi-analytical hybrid FEM with Sanders (1959) shell theory.

In order to solve the problem, Gorman (1976; 1982; 1995) used the superposition method of three problems with different boundary conditions. The solution is given in hyperbolic-trigonometric identities. The author used the idea of Timoshenko and Woinowsky-Krieger (1959), which they realized when solving the problem of a rectangular plate bending, with all edges clamped (superposition of two problems) and also when one edge is not clamped (superposition of three problems).

For the solution to the problem, Seok et al. (2004) applied a variational approach for approximating the transition from a $3 \mathrm{D}$ problem to a $2 \mathrm{D}$ one. They used exponential and trigonometric functions.

Looker and Sader (2008) obtained a simple analytic formula for natural frequencies calculation, using the variation principle and the singular perturbation theory. The approximating function consists of two items, one of which doesn't contain coordinates of $y$ and the second one contains $y^{2}$ as a multiplier. Numerical results are not available.

Zhong et al. (2013) used the double finite integral transform method for the vibration analysis. In the works by (Barton, 1951; Dalley and Ripperger, 1952; Lindholm et al., 1965; Leissa, 1969; Singal et al., 1992) experimental values of natural frequencies of the cantilever plate vibration were obtained. They are also given in Table 1.

The difference of the suggested numerical-analytical solution from the well-known solutions is that it can be checked by direct substitution into all equations of the given boundary-value problem of mathematical physics, which cannot be done with solutions in the form of arrays of numbers, which were obtained with numerical methods (FEM, FDM etc.). This method does not require solution superposition of simpler problems and is a direct, simpler and more reliable method that allows obtaining numerical results with any precision. When analyzing numerical series, it enables proving the presence of special points on the outline-the ends of the clamped edge-where bending moments are infinite. Variation methods usually "ignore" this fact.

\section{Method of Analysis}

\section{Mathematical Formulation}

Let us consider a rectangular cantilever Kirchhoff plate of constant thickness $h$. The relative proportions of the plate are $-\gamma / 2 \leq x \leq \gamma / 2,0 \leq y \leq 1$. The edge $y=0$ is clamped. Here $\gamma=a / b ; a \times b$ are plan proportions of the plate; $x=X / b, y=Y / b$ are relative coordinates of the plate's median surface.

The equation of free vibrations of the plate:

$\nabla^{2} \nabla^{2} W+\eta^{2} \frac{\partial^{2} W}{\partial t^{2}}=0$

where, $W(x, y, t)$-is the sought function of the median surface bendings, $t$ is time, $\nabla^{2}$-is the 2D Laplace operator; $\eta^{2}=\rho h b^{4} / D, \rho$-is the density of the plate's material, $D=E h^{3} /\left(12\left(1-v^{2}\right)\right)$ is the cylindrical rigidity of the plate, $E$ is Young's modulus, $v$ is Poisson's ratio.

According to the Fourier method, this function can be formulated as follows:

$W(x, y, t)=\left(C_{1} \cos p t+C_{2} \sin p t\right) w(x, y)$

Here, $C_{1}, C_{2}$ are arbitrary constants, which are determined from the initial conditions; $p$ is the vibration frequency (circular frequency) of the plate, which is to be determined; $w(x, y)$ is a coordinate function that must satisfy the differential equation:

$\nabla^{2} \nabla^{2} w(x, y)-\omega^{2} w(x, y)=0$

$\omega=p \eta=p b^{2} \sqrt{\rho h / D} \quad-$ is the natural relative vibration frequency. Function $w(x, y)$ determines mode shapes for the found frequency.

Then we will solve the problem of determining natural frequencies and mode shapes.

Boundary conditions of the problem (Timoshenko and Woinowsky-Krieger, 1959) are:

$w=0 ; \frac{\partial w}{\partial y}=0$ on the edge $y=0$

$\left\{\begin{array}{l}\frac{\partial^{2} w}{\partial y^{2}}+v \frac{\partial^{2} w}{\partial x^{2}}=0 \\ \frac{\partial^{3} w}{\partial y^{3}}+(2-v) \frac{\partial^{3} w}{\partial x^{2} \partial y}=0\end{array}\right.$ on the edge $y=1$

$\left\{\begin{array}{l}\frac{\partial^{2} w}{\partial x^{2}}+v \frac{\partial^{2} w}{\partial y^{2}}=0 ; \\ \frac{\partial^{3} w}{\partial x^{3}}+(2-v) \frac{\partial^{3} w}{\partial x \partial y^{2}}=0\end{array}\right.$ on the edges $x= \pm \gamma / 2$

The condition of the concentrated forces absence in free corner points is added to them:

$\frac{\partial^{2} w}{\partial x \partial y}=0$ with $x= \pm \gamma / 2, y=1$ 


\section{Symmetric Solution of the X-Coordinate}

Let us present the sought solution as a sum of three functions with undetermined ratios $A_{k}, B_{k}, C_{s}, D_{s}, E_{s}, F_{s}$, $R_{1}, R_{2}, R_{3}, R_{4}$ :

$$
\begin{aligned}
& w(x, y)=w_{1}(x, y)+w_{2}(x, y)+w_{3}(y) \\
& w_{1}(x, y)=\sum_{k=1,3}^{\infty}\left(A_{k} \cosh \alpha_{k} x+B_{k} \cosh \beta_{k} x\right) \sin \lambda_{k} y \\
& w_{2}(x, y) \\
& =\sum_{s=1}^{\infty}(-1)^{s}\left[\begin{array}{l}
C_{s} \sinh \left(\xi_{s}(y-1)\right)+D_{s} \sinh \left(\eta_{s}(y-1)\right) \\
+E_{s} \cosh \left(\xi_{s}(y-1)\right)+F_{s} \cosh \left(\eta_{s}(y-1)\right)
\end{array}\right] \cos \mu_{s} x \\
& w_{3}(y)=R_{1} \sinh \left(\omega_{*}(y-1)\right)+R_{2} \sin \left(\omega_{*}(y-1)\right) \\
& +R_{3} \cosh \left(\omega_{*}(y-1)\right)+R_{4} \cos \left(\omega_{*}(y-1)\right)
\end{aligned}
$$

where, $\lambda_{k}=\pi k / 2, \mu_{s}=2 \pi s / \gamma$.

Subordinating these functions to basic Equation 3, we will obtain:

$$
\begin{array}{ll}
\alpha_{k}=\sqrt{\lambda_{k}^{2}+\omega}, & \beta_{k}=\sqrt{\lambda_{k}^{2}-\omega}, \\
\xi_{s}=\sqrt{\mu_{s}^{2}+\omega}, & \eta_{s}=\sqrt{\mu_{s}^{2}-\omega}, \quad \omega_{*}=\sqrt{\omega}
\end{array}
$$

The introduction of the auxiliary function $w_{3}(y)$ is stipulated by the fact, that when satisfying the boundary conditions, the expansions of hyperbolic functions $\cosh \alpha_{k} x$ and $\cosh \beta_{k} x$ into series by $\cos \mu_{s} x$ will be used. These expansions contain constant terms that need compensating.

Functions Equation 9-11 satisfy boundary condition Equation 7. Let us demand that they satisfy all the remaining boundary conditions Equation 4-6. We will obtain a system of six equations:

$$
\begin{aligned}
& \sum_{s=1}^{\infty}(-1)^{s}\left(-C_{s} \sinh \xi_{s}-D_{s} \sinh \eta_{s}+E_{s} \cosh \xi_{s}\right. \\
& \left.\quad+F_{s} \cosh \eta_{s}\right) \cos \mu_{s} x-R_{1} \sinh \omega_{*}-R_{2} \sin \omega_{*} \\
& \quad+R_{3} \cosh \omega_{*}+R_{4} \cos \omega_{*}=0 \\
& \sum_{k=1,3, \ldots}^{\infty} \lambda_{k}\left(A_{k} \cosh \alpha_{k} x+B_{k} \cosh \beta_{k} x\right) \\
& \quad+\sum_{s=1}^{\infty}(-1)^{s}\left(C_{s} \xi_{s} \cosh \xi_{s}+D_{s} \eta_{s} \cosh \eta_{s}\right. \\
& \left.\quad-E_{s} \xi_{s} \sinh \xi_{s}-F_{s} \eta_{s} \sinh \eta_{s}\right) \cos \mu_{s} x \\
& \quad+\omega_{*}\left(R_{1} \cosh \omega_{*}+R_{2} \cos \omega_{*}-R_{3} \sinh \omega_{*}\right. \\
& \left.\quad+R_{4} \sin \omega_{*}\right)=0
\end{aligned}
$$

$$
\begin{aligned}
& \sum_{k=1,3, \ldots}^{\infty}(-1)^{\tilde{k}}\left[\begin{array}{l}
A_{k}\left(v \alpha_{k}^{2}-\lambda_{k}^{2}\right) \cosh \alpha_{k} x \\
+B_{k}\left(v \beta_{k}^{2}-\lambda_{k}^{2}\right) \cosh \beta_{k} x
\end{array}\right] \\
& -\sum_{s=1}^{\infty}(-1)^{s}\left[\begin{array}{c}
E_{s}\left(\xi_{s}^{2}-v \mu_{s}^{2}\right) \\
+F_{s}\left(\eta_{s}^{2}-v \mu_{s}^{2}\right)
\end{array}\right] \cos \mu_{s} x-\omega\left(R_{3}-R_{4}\right)=0 \\
& \sum_{s=1}^{\infty}(-1)^{s}\left\{\begin{array}{l}
C_{s}\left[\xi_{s}^{2}-(2-v) \mu_{s}^{2}\right] \xi_{s} \\
+D_{s}\left[\eta_{s}^{2}-(2-v) \mu_{s}^{2}\right] \eta_{s}
\end{array}\right\} \cos \mu_{s} x+\omega_{*}^{3}\left(R_{1}-R_{2}\right)=0 \\
& \sum_{k=1,3, \ldots}^{\infty}\left[A_{k}\left(\alpha_{k}^{2}-v \lambda_{k}^{2}\right) \cosh \tilde{\alpha}_{k}+B_{k}\left(\beta_{k}^{2}-v \lambda_{k}^{2}\right) \cosh \tilde{\beta}_{k}\right] \\
& \sin \lambda_{k} y+\sum_{s=1}^{\infty}\left[\begin{array}{l}
\left(v \xi_{s}^{2}-\mu_{s}^{2}\right)\left(\mathrm{C}_{s} \sinh \xi_{s} \tilde{y}+E_{s} \cosh \xi_{s} \tilde{y}\right) \\
+\left(v \eta_{s}^{2}-\mu_{s}^{2}\right)\left(D_{s} \sinh \eta_{s} \tilde{y}+F_{s} \cosh \eta_{s} \tilde{y}\right)
\end{array}\right] \\
& +\omega v\left[R_{1} \sinh \omega_{*} \tilde{y}-R_{2} \sin \omega_{*} \tilde{y}\right. \\
& \left.+R_{3} \cosh \omega_{*} \tilde{y}-R_{4} \cos \omega_{*} \tilde{y}\right]=0 \\
& \sum_{k=1,3, \ldots}^{\infty}\left\{\begin{array}{l}
A_{k}\left[\alpha_{k}^{2}-(2-v) \lambda_{k}^{2}\right] \alpha_{k} \sinh \tilde{\alpha}_{k} \\
+B_{k}\left[\beta_{k}^{2}-(2-v) \lambda_{k}^{2}\right] \beta_{k} \sinh \tilde{\beta}_{k}
\end{array}\right\} \sin \lambda_{k} y=0
\end{aligned}
$$

It is marked here:

$$
\tilde{\alpha}_{k}=\alpha_{k} \gamma / 2, \tilde{\beta}_{k}=\beta_{k} \gamma / 2, \tilde{k}=(k+1) / 2, \tilde{y}=y-1
$$

Hyperbolic functions of the $x$ argument, which are contained in Equation 14 and 15, expand into the Fourier series by $\cos \mu_{s} x$. These known expansions have a form of:

$\cosh \alpha_{k} x=\sinh \tilde{\alpha}_{k}\left[\frac{1}{\tilde{\alpha}_{k}}+\frac{4 \alpha_{k}}{\gamma} \sum_{s=1}^{\infty} \frac{(-1)^{s}}{\alpha_{k}^{2}+\mu_{s}^{2}} \cos \mu_{s} x\right]$

The expansion of the function $\cosh \tilde{\beta}_{k} x$ is obtained after replacing $\alpha_{k}$ with $\beta_{k}$ and $\tilde{\alpha}_{k}$ with $\tilde{\beta}_{k}$.

Expansions of Equation 19 contain constant terms which are added to free items from the auxiliary function Equation 11.

Then, functions of the $y$ argument, which appear in Equation 17, expand into the Fourier series by $\sin \lambda_{k} y$ :

$$
\begin{aligned}
& \sinh \xi_{s} \tilde{y}=-2 \sum_{k=1,3, \ldots}^{\infty} \frac{(-1)^{\tilde{k}} \xi_{s}+\lambda_{k} \sinh \xi_{s}}{\lambda_{k}^{2}+\xi_{s}^{2}} \sin \lambda_{k} y \\
& \sin \omega_{*} \tilde{y}=-2 \sum_{k=1,3, \ldots}^{\infty} \frac{(-1)^{\tilde{k}} \omega_{*}+\lambda_{k} \sin \omega_{*}}{\lambda_{k}^{2}-\omega} \sin \lambda_{k} y \\
& \cosh \xi_{s} \tilde{y}=2 \cosh \xi_{s} \sum_{k=1,3, \ldots}^{\infty} \frac{\lambda_{k}}{\lambda_{k}^{2}+\xi_{s}^{2}} \sin \lambda_{k} y \\
& \cos \omega_{*} \tilde{y}=2 \cos \omega_{*} \sum_{k=1,3, . .}^{\infty} \frac{\lambda_{k}}{\lambda_{k}^{2}-\omega} \sin \lambda_{k} y
\end{aligned}
$$


The remaining expansions are obtained by replacing $\xi_{s}$ with $\eta_{s}$ or $\omega_{s}$.

Demanding that in Equation 13-16 the combinations of free items turn into zero, we will obtain a separate auxiliary subsystem of four equations for ratios $R_{1}, R_{2}$, $R_{3}, R_{4}$, the solution to which is:

$R_{1}=-\frac{G \omega_{*}\left(\cosh \omega_{*}+\cos \omega_{*}\right)}{2 \omega\left(1+\cosh \omega_{*} \cos \omega_{*}\right)}$

$+\frac{H\left(\sinh \omega_{*} \cos \omega_{*}+\cosh \omega_{*} \sin \omega_{*}\right)}{2 \omega\left(1+\cosh \omega_{*} \cos \omega_{*}\right)}, \quad R_{2}=R_{1}$

$R_{3}=-\frac{G \omega_{*}\left(\sinh \omega_{*}+\sin \omega_{*}\right)}{2 \omega\left(1+\cosh \omega_{*} \cos \omega_{*}\right)}$

$+\frac{H\left(1+\sinh \omega_{*} \sin \omega_{*}+\cosh \omega_{*} \cos \omega_{*}\right)}{2 \omega\left(1+\cosh \omega_{*} \cos \omega_{*}\right)}, R_{4}=R_{3}-\frac{H}{\omega}$

Here:

$G=\frac{2}{\gamma} \sum_{k=1,3, \ldots}^{\infty} \lambda_{k}\left(\frac{A_{k}}{\alpha_{k}} \sinh \tilde{\alpha}_{k}+\frac{B_{k}}{\beta_{k}} \sinh \tilde{\beta}_{k}\right)$

$H=\frac{2}{\gamma} \sum_{k=1,3, \ldots}^{\infty}(-1)^{\tilde{k}}\left(\begin{array}{l}\frac{A_{k}}{\alpha_{k}}\left(v \alpha_{k}^{2}-\lambda_{k}^{2}\right) \sinh \tilde{\alpha}_{k} \\ +\frac{B_{k}}{\beta_{k}}\left(v \beta_{k}^{2}-\lambda_{k}^{2}\right) \sinh \tilde{\beta}_{k}\end{array}\right)$

In the remaining system, inner expressions of trigonometric series must turn into zero. As a result, we obtain the basic system of $6 \times \infty$ equations:

$$
\begin{aligned}
& -C_{s} \operatorname{sh} \xi_{s}-D_{s} \operatorname{sh} \eta_{s}+E_{s} \operatorname{ch} \xi_{s}+F_{s} \operatorname{ch} \eta_{s}=0 \\
& C_{s} \xi_{s} \operatorname{ch} \xi_{s}+D_{s} \eta_{s} \operatorname{ch} \eta_{s}-E_{s} \xi_{s} \operatorname{sh} \xi_{s}-F_{s} \eta_{s} \operatorname{sh} \eta_{s}=S_{1 s} \\
& E_{s}\left(\xi_{s}^{2}-v \mu_{s}^{2}\right)+F_{s}\left(\eta_{s}^{2}-v \mu_{s}^{2}\right) F_{s}=S_{2 s}
\end{aligned}
$$

$$
\begin{aligned}
& C_{s}\left[\xi_{s}^{2}-(2-v) \mu_{s}^{2}\right] \xi_{s}+D_{s}\left[\eta_{s}-(2-v) \mu_{s}^{2}\right] \eta_{s}=0 \\
& A_{k}\left(\alpha_{k}^{2}-v \lambda_{k}^{2}\right) \operatorname{ch} \tilde{\alpha}_{k}+B_{k}\left(\beta_{k}^{2}-v \lambda_{k}^{2}\right) \operatorname{ch} \tilde{\beta}_{k} \\
& +2 \sum_{s=1}^{\infty}\left\{\begin{array}{l}
-C_{s} \frac{v \xi_{s}^{2}-\mu_{s}^{2}}{\lambda_{k}^{2}+\xi_{s}^{2}}\left[(-1)^{\tilde{k}} \xi_{s}+\lambda_{k} \operatorname{sh} \xi_{s}\right] \\
-D_{s} \frac{v \eta_{s}^{2}-\mu_{s}^{2}}{\lambda_{k}^{2}+\eta_{s}^{2}}\left[(-1)^{\tilde{k}} \eta_{s}+\lambda_{k} \operatorname{sh} \eta_{s}\right] \\
+E_{s} \frac{v \xi_{s}^{2}-\mu_{s}^{2}}{\lambda_{k}^{2}+\xi_{s}^{2}} \lambda_{k} \operatorname{ch} \xi_{s}+F_{s} \frac{v \eta_{s}^{2}-\mu_{s}^{2}}{\lambda_{k}^{2}+\eta_{s}^{2}} \lambda_{k} \operatorname{ch} \eta_{s}
\end{array}\right\} \\
& +2 v \omega\left\{\begin{array}{l}
R_{1}\left[(-1)^{\tilde{k}} \omega_{*}+\lambda_{k} \operatorname{sh} \omega_{*}\right] \\
\lambda_{k}^{2}+\omega \\
+\frac{R_{2}}{\lambda_{k}^{2}-\omega}\left((-1)^{\tilde{k}} \omega_{*}+\lambda_{k} \sin \omega_{*}\right) \\
+R_{3} \frac{\lambda_{k} \operatorname{ch} \omega_{*}}{\lambda_{k}^{2}+\omega}-R_{4} \frac{\lambda_{k} \cos \omega_{*}}{\lambda_{k}^{2}-\omega}
\end{array}\right\}=0
\end{aligned}
$$

$$
\begin{aligned}
& A_{k}\left[\alpha_{k}^{2}-(2-v) \lambda_{k}^{2}\right] \alpha_{k} \operatorname{sh} \tilde{\alpha}_{k} \\
& +B_{k}\left[\beta_{k}^{2}-(2-v) \lambda_{k}^{2}\right] \beta_{k} \operatorname{sh} \tilde{\beta}_{k}=0
\end{aligned}
$$

Where:

$$
\begin{gathered}
S_{1 s}=-\frac{4}{\gamma} \sum_{k=1,3, \ldots}^{\infty} \lambda_{k}\left(A_{k} \frac{\alpha_{k} \sinh \tilde{\alpha}_{k}}{\alpha_{k}^{2}+\mu_{s}^{2}}+B_{k} \frac{\beta_{k} \sinh \tilde{\beta}_{k}}{\beta_{k}^{2}+\mu_{s}^{2}}\right) \\
S_{2 s}=\frac{4}{\gamma} \sum_{k=1,3, \ldots}^{\infty}(-1)^{\tilde{k}}\left[\begin{array}{c}
A_{k} \frac{v \alpha_{k}^{2}-\lambda_{k}^{2}}{\alpha_{k}^{2}+\mu_{s}^{2}} \alpha_{k} \sinh \tilde{\alpha}_{k} \\
+B_{k} \frac{v \beta_{k}^{2}-\lambda_{k}^{2}}{\beta_{k}^{2}+\mu_{s}^{2}} \beta_{k} \sinh \tilde{\beta}_{k}
\end{array}\right]
\end{gathered}
$$

The basic subsystem of six equations for ratios $A_{k}, B_{k}$, $C_{s}, D_{s}, E_{s}, F_{s}$ was then reduced to one equation (to one infinite system, to be more precise) relative to the ratios $A_{k}(k=1,3, \ldots)$ :

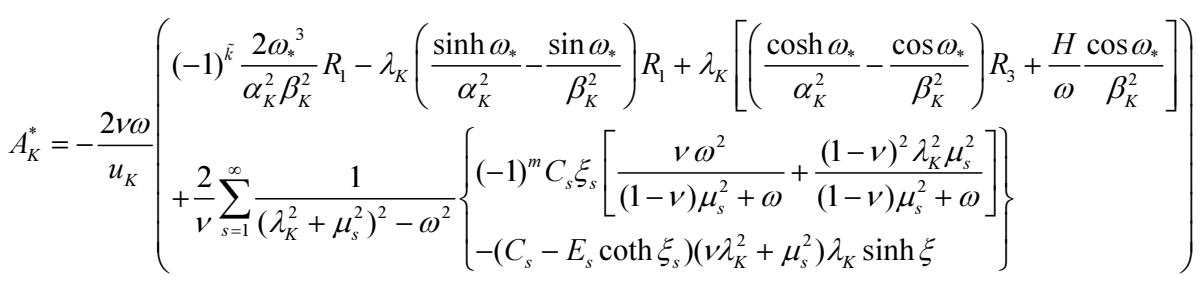

where, marked $A_{K}^{*}=A_{K} \sinh \tilde{\alpha}_{K}$ :

$$
\begin{aligned}
& u_{K}=\frac{\beta_{K}\left[(1-v) \lambda_{K}^{2}+\omega\right]^{2} \operatorname{coth} \tilde{\alpha}_{K}}{\beta_{K}\left[(1-v) \lambda_{K}^{2}+\omega\right]}+ \\
& +\frac{\alpha_{K}\left[(1-v) \lambda_{K}^{2}-\omega\right]^{2} \operatorname{coth} \tilde{\beta}_{K}}{\beta_{K}\left[(1-v) \lambda_{K}^{2}+\omega\right]}
\end{aligned}
$$

In order not to confuse indices $k$ in and out the internal sums, the external index is designated as $K$. To prevent the system Equation 36 from appearing bulky, the ratios $C_{s}$ and $E_{s}$, that are contained in it, are not included into this expression. They were determined based on a separate system of two linear algebraic equations and then were substituted into Equation 36 in computing: 


$$
\left\{\begin{array}{l}
C_{s} \delta_{s}\left(\frac{\sinh \xi_{s}}{\cosh \eta_{s}}-\frac{\delta_{s} \xi_{s}}{\eta_{s}} \tanh \eta_{s}\right) \\
-E_{s}\left(\delta_{s} \frac{\cosh \xi_{s}}{\cosh \eta_{s}}-1\right)=\frac{S_{2 S}}{(1-v) \mu_{s}^{2}+\omega} \\
C_{s}\left(\xi_{s} \cosh \xi_{s}-\eta_{s} \tanh \eta_{s} \sinh \xi_{s}-\frac{\delta_{s} \xi_{s}}{\cosh \eta_{s}}\right) \\
-E_{s}\left(\xi_{s} \sinh \xi_{s}-\eta_{s} \tanh \eta_{s} \cosh \xi_{s}\right)=\mathrm{S}_{1 S}
\end{array}\right.
$$

$$
\text { where } \delta_{s}=\left[(1-v) \mu_{s}^{2}-\omega\right] /\left[(1-v) \mu_{s}^{2}+\omega\right] \text {. }
$$

Ratios $B_{k}$ are expressed through $A_{k}$ from Equation 33. Ratios $D_{s}$ and $F_{s}$ are expressed through "basic" ratios $C_{s}$ and $E_{s}$ from Equation 30 and 31 and those after solving the system Equation 38 through ratios $A_{k}$ from Equation 34, 35 and 32. Therefore the expression Equation 36 is an infinite homogeneous system of linear algebraic equations relative to one sequence of $A_{K}^{*}$, which can be briefly put as follows:

$$
A_{K}^{*}=\sum_{k=1,3, \ldots}^{\infty} b_{K k} A_{k}^{*}(K=1,3 \ldots)
$$

The basic system Equation 36 also contains an unknown frequency $\omega$.

The determinant of the system Equation 36 must be equal to zero for it to have non-trivial solutions. If we make and expand the determinant, we will obtain a frequency equation, the solution to which will give a spectrum of natural frequencies. In this case, obtaining a transcendental frequency equation in the expanded form and its solution is quite a difficult mathematical problem. Here it is suggested to use a very simple method of obtaining natural frequencies.

We will calculate the ratios $A_{k}^{*}$ in the right part of the system Equation 39 with the previous iteration and similar ratios $A_{k}^{*}$ in the left part with the next iteration.

It is necessary to select such values of $\omega_{i}$, with which the method of successive approximations, organized by Equation 36, would lead at some iteration to the equations $A_{k(N+1)}^{*}=A_{k N}^{*} \neq 0$ for all subsequent values of $N$. These would be the non-trivial solutions of the homogeneous system. As the zeroth-order approximation, we can put all $A_{k}^{*}=1$. The frequencies, found with this method, would be the sought frequencies of free vibrations and the found ratios $A_{k N}^{*}$ (with the accuracy of the multiplier, constant to all $A_{k N}^{*}$ ) would give the corresponding mode shapes by substituting into Equation 8. The equation $A_{k(N+1)}^{*}=A_{k N}^{*} \neq 0$ means that vibrations occur with constant amplitude, i.e., they are free and undamped. Undoubtedly, finding the natural frequencies of $\omega_{i}$ will take certain time, but it is not hard to choose an optimal strategy for the parameter $\omega$ exhaustion.

\section{Antisymmetric Solution of the X-Coordinate}

Apart from the mode shapes, symmetric about $O y$ axis, there must be antisymmetric ones.

The sought coordinate function of bendings now will look like:

$$
\begin{aligned}
w(x, y) & =w_{1}(x, y)+w_{2}(x, y) \\
w_{1}(x, y) & =\sum_{k=1,3, \ldots}^{\infty}\left(A_{k} \sinh \alpha_{k} x+B_{k} \sinh \beta_{k} x\right) \sin \lambda_{k} y \\
w_{2}(x, y) & =\sum_{s=1,3, \ldots}^{\infty}(-1)^{(s+1) / 2}\left[C_{s} \sinh \left(\xi_{s}(y-1)\right)\right. \\
& +D_{s} \sinh \left(\eta_{s}(y-1)\right)+E_{s} \cosh \left(\xi_{s}(y-1)\right) \\
& \left.+F_{s} \cosh \left(\eta_{s}(y-1)\right)\right] \sin \mu_{s} x
\end{aligned}
$$

Instead of even functions with $x$, there are odd functions in it with no additional item $w_{3}$. The ratios of the series $A_{k}, B_{k}, C_{s}, D_{s}, E_{s}, F_{s}$ are designated with the same letters for convenience, although they are different from the even-type solution. The ratios: $\alpha_{k}, \beta_{k}, \xi_{s}, \eta_{s}, \lambda_{k}$ $E_{s}$ are the same, but here $\mu_{s}=\pi s / \gamma$, in contrast to the even-type solution.

Satisfying the boundary conditions of the problem, we will obtain a system of six equations, which we will be reduced to one equation:

$$
\begin{aligned}
& A_{K}^{*}=\frac{4 \omega}{u_{K}} \\
& \times \sum_{s=1,3, \ldots}^{\infty} \frac{1}{\left(\lambda_{K}^{2}+\mu_{s}^{2}\right)^{2}-\omega^{2}}\left\{\begin{array}{c}
(-1)^{m} C_{s} \xi_{s}\left[\begin{array}{c}
\frac{v \omega^{2}}{(1-v) \mu_{s}^{2}+\omega} \\
+\frac{(1-v)^{2} \lambda_{K}^{2} \mu_{s}^{2}}{(1-v) \mu_{s}^{2}+\omega}
\end{array}\right] \\
-\left(C_{s}-E_{s} \operatorname{coth} \xi_{s}\right)\left(v \lambda_{K}^{2}+\mu_{K}^{2}\right) \lambda_{K} \sinh \xi
\end{array}\right\}
\end{aligned}
$$

Let us note that this expression only differs from the series in Equation 36 in the sign and also in odd indices s. In Equation 43 (compare with Equation 37):

$$
\begin{aligned}
& A_{K}^{*}=A_{K} \cosh \tilde{\alpha}_{K}, \\
& u_{K}=\frac{\beta_{K}\left[(1-v) \lambda_{K}^{2}+\omega\right]^{2} \tanh \tilde{\alpha}_{K}}{\beta_{K}\left[(1-v) \lambda_{K}^{2}+\omega\right]} \\
& +\frac{\alpha_{K}\left[(1-v) \lambda_{K}^{2}-\omega\right]^{2} \tanh \tilde{\beta}_{K}}{\beta_{K}\left[(1-v) \lambda_{K}^{2}+\omega\right]}
\end{aligned}
$$

Let us list (in order not to write formulae) the rest of similarities and differences. The ratios $C_{s}$ and $E_{s}$ are determined from the system that coincides with Equation 38. In Equation 33 for $B_{k}$ hyperbolic sines are replaced with hyperbolic cosines. In the expressions for $S_{1 s}$ and 
$S_{2 s}$ Equation 34 and35, except for this replacement, their signs will also change.

\section{Results and Discussion}

There was developed a program in the system of analytical calculations Maple to calculate natural frequencies and to obtain the corresponding mode shapes. A square plate was considered as an example; the Poisson number was equal to 0.3 . From 39 to 99 items were kept in the series (this and the size of the reduced resolution systems Equation 36 and 43). The number of iterations is from 20-180. The word length is $30-500$ characters. The frequency band $0<\omega<65$ was studied.

The first seven of the found frequencies are given in the first line of Table 1 . The results, obtained by other researchers, are given in the next lines for comparison. Two lines of the results of Bazley et al. (1967) in the table are upper and lower boundaries of frequencies correspondingly. Let us note, that the results of Gorman (1976) are obtained with $v=0.333$.

Frequencies of the symmetric vibrations $\omega_{1}, \omega_{4}, \omega_{6}$ in this study were obtained keeping 99 items in the series. It took 20 iterations for the coincidence of five significant figures. The calculations were made with 300 significant figures.

The satisfying value of the frequency $\omega_{3}=21.284$ was only obtained after a considerable increase in the number of iterations up to 180 . In the series 59 items were kept. Because of the bad convergence of the process around this value and the accumulation of errors, the rise in the number of items in the series ( 99 and more) led to a computing failure even after increasing the number of significant figures in calculations (up to 500). We cannot explain this fact for now.

Odd frequencies were found keeping 99 items in the series. It took 20 iterations for the frequencies $\omega_{2}$ and $\omega_{5}$. It took 50 iterations for the frequency $\omega_{8}$. The graphs of the corresponding mode shapes were obtained for the found frequencies. They are shown in Fig. 1-7.

Using the developed programs, it is not hard to obtain even higher natural frequencies of cantilever plate vibrations for different ratios of the plate's sides.

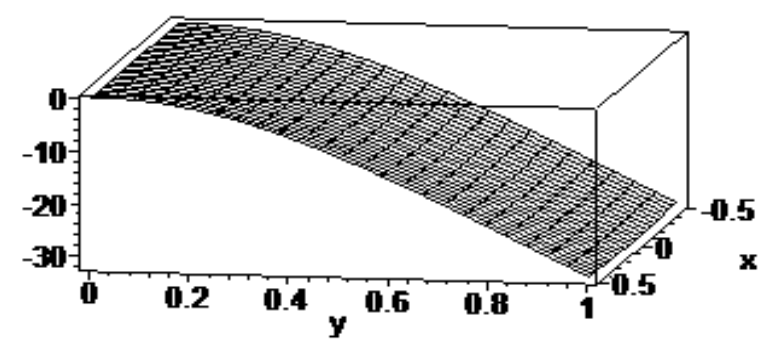

Fig. 1. The first even free vibration mode shape of the square cantilever plate $(\omega=3.471)$

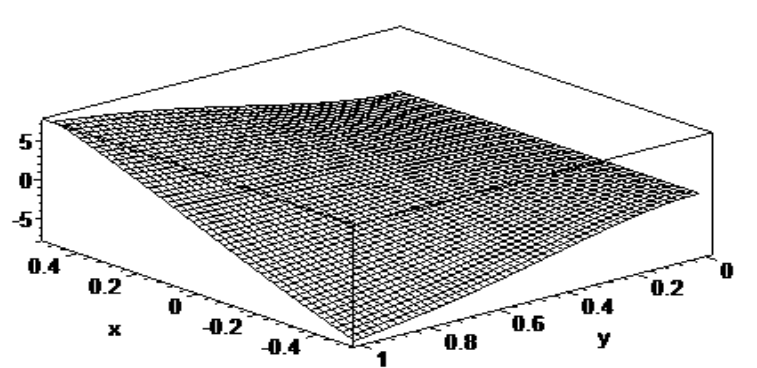

Fig. 2. The first odd free vibration mode shape of the square cantilever plate $(\omega=8.506)$

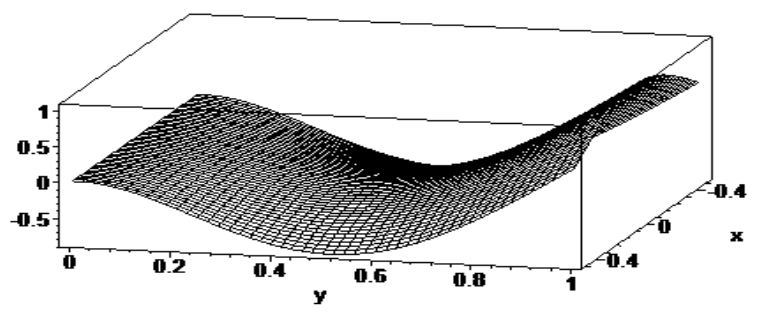

Fig. 3. The second even free vibration mode shape of the square cantilever plate $(\omega=21.284)$

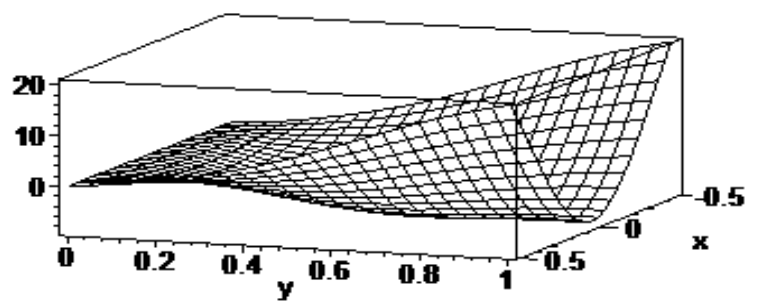

Fig. 4. The third even free vibration mode shape of the square cantilever plate $(\omega=27.199)$

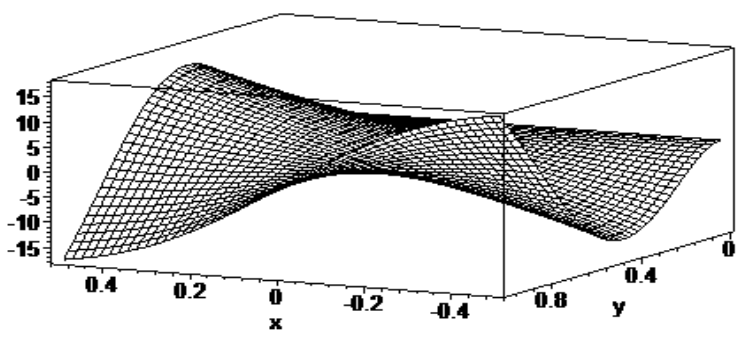

Fig. 5. The second odd free vibration mode shape of the square cantilever plate $(\omega=30.954)$

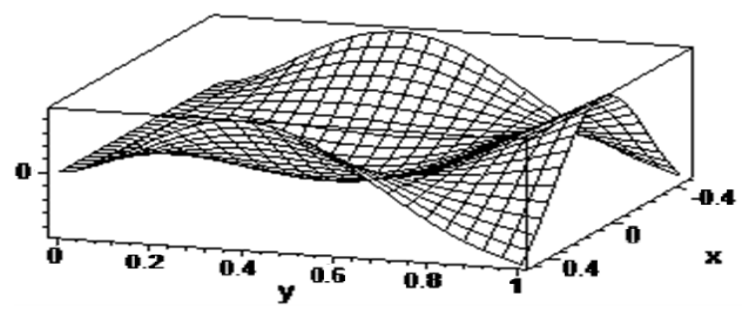

Fig. 6. The fourth even free vibration mode shape of the square cantilever plate $(\omega=54.184)$ 


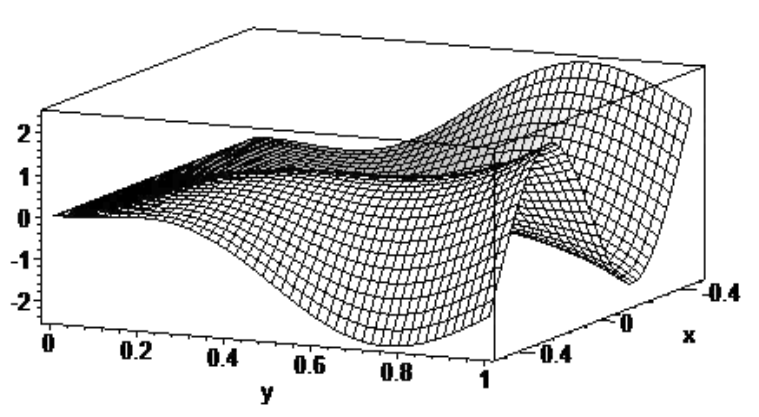

Fig. 7. The third odd free vibration mode shape of the square cantilever plate $(\omega=64.143)$

The comparison with other authors' results shows that the results obtained by (Young, 1950; Barton, 1951) with the Ritz method are too high. The experimental data of Barton (1951; Dalley and Ripperger, 1952; Lindholm et al., 1965; Leissa, 1969) and also Singal et al. (1992) confirm the same. The results of Leissa (1969) are closer (but also too high). Let us note that we did not find the frequency similar to $\omega_{7}$ in the works of Gorman (1976; 1982; 1995; Rossi and Laura, 1996) despite the detailed study of frequencies close to this value. The results of Seok et al. (2004; Kerboua et al., 2007; Bardell, 1991), who found the first four frequencies, are the closest to the results obtained in this study. The numerical results in the work of Rossi and Laura (1996) are almost matching with the ones in the given paper. First two frequencies with the results of Gorman (1976) are also similar. He calculated them separately for $v=0.3$. Unfortunately, other frequencies in (1976) for $v=0.3$ were not calculated (except for $\omega_{7}$ and $\omega_{9}$, which we can compare with nothing). Let us note that in the works by $\mathrm{Wu}$ et al. (2009; Fu and Price, 1987) the frequencies are given in Hz. In this study they are recalculated in nondimensional quantities. The non-dimensional frequencies in the work by Claassen and Thorne (1962), in the research by Seok et al. (2004), in the Eftekhari and Jafari (2013), in the Shi et al. (2014) were multiplied by $\pi^{2}$ in order to bring them in line with the formula for relative frequencies in Table 1.

Note that all the natural frequencies obtained by Eftekhari and Jafari (2013; Shi et al., 2014) for the plate of constant thickness Mindlin are slightly less than the corresponding values of the Kirchhoff plate, with a more marked difference in the overtones.

The numerical results in this study are in better agreement with the experimental data (Barton, 1951; Dalley and Ripperger, 1952; Lindholm et al., 1965; Leissa, 1969; Singal et al., 1992) than the results of some other authors. Besides, it is worth mentioning that the difficulties in maintaining the perfect clamping in experiments cause too low results for natural frequencies. In the work of Lindholm et al. (1965; Singal et al., 1992) the natural frequencies are given in $\mathrm{Hz}$. They are recalculated in non-dimensional quantities and given in Table 1. Let us note that in the paper by Singal et al. (1992) the experiment was conducted with aluminum plates. The value of the Poisson's ratio is not given (0.320.36 according to the reference-book). It is hard to explain why the natural frequencies in the study by Singal et al. (1992) turned out the least among all other corresponding experimental values (Barton, 1951; Dalley and Ripperger, 1952; Lindholm et al., 1965; Leissa, 1969).

Mizusawa (1986; Bardell, 1991; Rossi and Laura, 1996) researched into the effect of Poisson's ratio on values of natural frequencies. Mizusawa (1986) calculated the natural frequencies (the first five of them) with the values of Poisson's ratio $v=0,0.15,0.3$ and 0.5 . With the increase in this ratio, the decrease of the corresponding natural frequencies was observed. For instance, the first frequency of a square plate adopted the next values: 3.509, 3.499, 3.467 and 3.373. Bardell (1991) obtained a graphical dependency of the frequencies on Poisson's ratio. The highest frequencies also decreased with the increase in Poisson's ratio; the first frequency remained constant according to the graph, apparently due to a large scale. Unfortunately, there are no numerical results in this study. Rossi and Laura (1996) calculated the first 10 frequencies for the values of Poisson's ratio from 0 to 0.5 with 0.1 step. In this connection, the corresponding values of the frequencies decreased.

For comparison, the frequencies $\omega_{1}, \omega_{2}$ and $\omega_{4}$ were calculated with $v=0.333$ according to the given theory. They worked out 3.4596, 8.3560 and 27.0645 (other frequencies were not calculated). These values coincide very accurately with the results of Rossi and Laura (1996; Gorman, 1976) and confirm the decrease of frequencies when Poisson's ratio increases.

Earlier it was noted that all ratios of the initial estimate in solving the systems Equation 36 and 43 set equal to one $A_{k}^{*}=1$. Since these systems are linear and homogeneous, all ratios of the series were calculated up to the precision of a constant. The same applies to the obtained mode shapes. Some researchers noted that the first mode shape (Fig. 1) is similar to the shape of the bent plate under the influence of a uniform transverse load. Selecting other initial values of $A^{*}{ }_{k}$ (with the first natural frequency $\omega=3.471$ ), we were able to determine that if we assume all $A_{k}^{*}=0.004025$, the iteration process led almost accurately to the shape of the bent surface of the square cantilever plate under the influence of a uniform transverse load.

Table 2 presents bendings of the free edge opposing the clamped one and the bending moments $M_{y}$ in the clamped section of the square cantilever plate in static bending by a uniform unit load and in the first free vibration mode shape. The $w$ bending is related to the value $q_{0} b^{4} / D$ and the bending moments $M_{y}$ is to the value, where $q_{0}$ is a uniform transverse load. The values of the bendings and the moments of static bending were 
obtained by the authors using the iterative method of hyperbola-trigonometric series superposition. Thus the divergence of the numerical series $M_{y}$ was established at the ends of the clamped edge, where near these points the bending moments tend to $-\infty$. Therefore, the ends of the clamped edge are stress concentrators due to the drastic change of the boundary conditions. If 0.5 is added to the values of the bending moments of the first mode shape, we will obtain $0.53467 ; 0.53441 ; 0.53292$; $0.52646 ; 0.49666 ; 0.39856$ respectively. The first five values in bending and vibration are in line with each other. The last value in the corner point naturally has a finite value after the summation of the finite series, although calculations after the increase in the number of members in the series (the increase in the size of the reduced system) showed its absolute increase, whereas the values in the internal points of the clamped edge did not change up to the sixth significant digit.

Free vibrations of the plate can be produced differently, e.g., with impact loads, the termination of concentrated or distributed transverse loads etc. In the general case, first of all, there will be a transient vibration process, which is determined by the nature of the initial conditions of the problem. This process is followed by the steady-state vibration with a full spectrum of natural mode shapes and frequencies (if no damping). If the plate was initially bent by a uniform load and then the load is removed, there will be no transient process and the plate will immediately get into the mode of steady-state vibration. When the load is removed, the dynamic bending moments will decrease by the value of the even moment 0.5 in the cylindrical bending.

Table 2. Values of the free edge $y=1$ bendings and the bending moments of the clamped edge of the square plate while keeping 79 items in the series under bending and vibration

\begin{tabular}{lllll}
\hline & & {$\left[M_{y}\right]_{y=0}$} & {$[W]_{y-1}$} & {$\left[M_{y}\right]_{y=0}$} \\
$x$ & Bending & Bending & $\omega_{1}=3.471$ & $\omega_{1}=3.471$ \\
\hline 0 & -0.12907 & 0.5302 & -0.12937 & 0.03467 \\
0.1 & -0.12899 & 0.5302 & -0.12927 & 0.03441 \\
0.2 & -0.12875 & 0.52959 & -0.12898 & 0.03292 \\
0.3 & -0.12835 & 0.52512 & -0.12851 & 0.02646 \\
0.4 & -0.12783 & 0.49938 & -0.12788 & -0.00334 \\
0.5 & -0.12724 & $-\infty$ & -0.12716 & -0.60144 \\
\hline
\end{tabular}

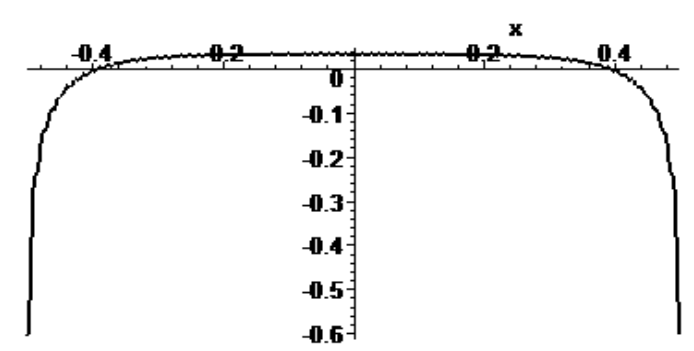

Fig. 8. The dynamical bending moments $M_{y}$ in the clamped edge at $\omega=3.471$
Figure 8 shows the epure of dynamic bending moments in the clamped section of the square plate with the first frequency and the largest vibration amplitude.

\section{Conclusion}

This paper offers an effective method of determining natural frequencies and mode shapes of a rectangular cantilever Kirchhoff plate using two trigonometric series, which contain hyperbolic functions by another coordinate. The problem of finding natural frequencies is brought to simple exhaustion of frequencies in a resolution homogeneous reduced system of linear algebraic equations relative to one sequence of trigonometric series ratios. The frequency was taken as a natural frequency, at which the given ratios of two neighbor iterations did not differ from each other, ensuring the equality to zero of the system determinant and its non-trivial solution. The obtained natural frequencies are remarkable for high accuracy and correlate with the results of other authors who solved this problem with different methods of high accuracy meeting all the problem's conditions. The difference of this method from others is that an analytically obtained accurate solution is an infinite system of linear equations relative to ratios of hyperbolic-trigonometric series, which contains vibration frequency as a parameter. The obtained natural frequencies and series ratios can be checked by forward substitution into all the problem's equations. It is not hard to enlarge the reduced system and increase the calculation accuracy in the numerical implementation.

It is demonstrated that the first natural frequency gives a mode shape, which corresponds with the cantilever plate's bending under the influence of a uniform transverse load. It is also determined that ends of the clamped edge are special points, in which bending moments are infinite, therefore tensions are also infinite.

The given method can also be applied to a plate, all edges of which are clamped and also at other combinations of clamped and free edges, including Reissner-Timoshenko plates.

\section{Acknowledgment}

The authors would like to gratefully acknowledge the unknown reviewers for their review and helpful comments.

\section{Author's Contributions}

The authors have equally contributed to the conducted research and writing of the article.

\section{Ethics}

This article is original and contains unpublished material. The authors confirm that there are no ethical issues involved. 


\section{References}

Anderson, R.G., B.M. Irons and O.C. Zienkiewicz, 1968. Vibration and stability of plates using finite elements. Int. J. Solids Structures, 4: 1031-1055. DOI: 10.1016/0020-7683(68)90021-8

Bardell, N.S., 1991. Free vibration analysis of a flat plate using the hierarchical finite element method. J. Sound Vibrat., 151: 263-289.

DOI: $10.1016 / 0022-460 x(91) 90855-E$

Barton, M.V., 1951. Vibration of rectangular and skew cantilever plates. J. Applied Mechan., 18: 129-134.

Bazley, N.W., D.W. Fox and J.T. Stadter, 1967. Upper and lower bounds for the frequencies of rectangular cantilever plates. J. Applied Mathemat. Mechan., 47: 251-260. DOI: 10.1002/zamm.19670470406

Claassen, R.W. and C.J. Thorne, 1962. Vibration of a rectangular cantilever plate. J. Aerospace Sci., 29: 1300-1305. DOI: $10.2514 / 8.9800$

Courant, R., 1943. Variational methods for the solution of problems of equilibrium and vibrations. Bull. Am. Math. Society, 49: 1-23. DOI: 10.1090/S0002-9904-1943-07818-4

Dalley, I.W. and E.A. Ripperger, 1952. Experimental values of natural frequencies for skew and rectangular cantilever plates. Proce. Society Experim. Stress Analysis, 9: 51-66.

Eftekhari, S.A. and A.A. Jafari, 2013. A simple and accurate Ritz formulation for free vibration of thick rectangular and skew plates with general boundary conditions. Acta Mech., 224: 193-209.

DOI: $10.1007 / \mathrm{s} 00707-012-0737-6$

$\mathrm{Fu}$, Y. and W.G. Price, 1987. Interactions between a partially or totally immersed vibrating cantilever plate and the surrounding fluid. J. Sound Vibrat., 118: 495-513. DOI: 10.1016/0022-460X(87)90366-X

Gorman, D.J., 1976. Free vibration analysis of cantilever plates by the method of superposition. J. Sound Vibrat., 49: 453-467.

DOI: $10.1016 / 0022-460 X(76) 90828-2$

Gorman, D.J., 1982. Free vibration analysis of rectangular plates. Amsterdam: Elsevier. DOI: 10.1016/0022-460X(82)90329-7

Gorman, D.J., 1995. Accurate free vibration analysis of the orthotropic cantilever plate. J. Sound Vibrat., 181: 605-618. DOI: 10.1006/JSVI.1995.0161

Kerboua, Y., A.A. Lakis, M. Thomas and L. Marcouiller, 2007. Hybrid method for vibration analysis of rectangular plates. Nuclear Eng. Des., 237: 791-801.

DOI: $10.1016 /$ j.nucengdes.2006.09.025

Leissa A.W., 1973. The free vibration of rectangular plates. J. Sound Vibrat., 31: 257-293. DOI: $10.1016 / \mathrm{S} 0022-460 x(73) 80371-2$

Leissa, A.W., 1969. Vibration of Plates. 1st Edn., Scientific and Technical Information Division, National Aeronautics and Space Administration, pp: 353.
Liew, K.M., K.Y. Lam and S.T. Chow, 1990. Free vibration analysis of rectangular plate using orthogonal plate function. Comput. Structures, 34: 79-85. DOI: 10.1016/0045-7949(90)90302-I

Lindholm, U.S., D.D. Kana, W.H. Chu and H.N. Abramson, 1965. Elastic vibration characteristics of cantilever plates in water. J. Ship Res., 9: 11-22.

Looker, J.R. and J.E. Sader, 2008. Flexural resonant frequencies of thin rectangular cantilever plates. J. Applied Mechan., 75: 011007-1-8. DOI: $10.1115 / 1.2745377$

Martin, A.I., 1956. On the vibration of cantilever plate. Q. J. Mechan. Applied Mathemat., 9: 94-102. DOI: $10.1093 /$ qjmam/9.1.94

Mizusawa, T., 1986. Natural frequencies of rectangular plates with free edges. J. Sound Vibrat., 105: 451- 459. DOI: 10.1016/0022-460X(86) 90171-9

Rossi, R.E. and P.A.A. Laura, 1996. Symmetric and antisymmetric normal modes of a cantilever rectangular plate: Effect of Poisson's ratio and a concentrated mass. J. Sound Vibrat., 195: 142-148. DOI: 10.1006/JSVI.1996.0410

Sanders, J.L., 1959. An improved first approximation theory for thin shell. NASA TR-24.

Seok, J., H.F. Tiersten and H.A. Scarton, 2004. Free vibrations of rectangular cantilever plates. Part 2: Inplane motion. J. Sound Vibrat., 271: 147-158. DOI: $10.1016 / \mathrm{S} 0022-460 \mathrm{X}(03) 00365-1$

Shi, D., Q. Wang, X. Shi and F. Pang, 2014. Free Vibration analysis of moderately thick rectangular plates with variable thickness and arbitrary boundary conditions. Shock Vibrat. DOI: $10.1155 / 2014 / 572395$

Singal, R.K., D.J. Gorman and S.A. Forgues, 1992. A comprehensive analytical solution for free vibration of rectangular plates with classical edge conditions: Experimental verification. Experim. Mechan., 32: 21-23. DOI: 10.1007/BF02317979

Timoshenko, S. and S. Woinowsky-Krieger, 1959. Theory of Plates and Shells. 2nd Edn., McGrawHill, New York, pp: 580.

Warburton, G.B., 1954. The vibration of rectangular plates. Proc. Institute Mechan. Eng., 168: 371-384. DOI: 10.1243/PIME_PROC_1954_168_040_02

Wu, Z., X. Ma, P.N. Brett and J. Xu, 2009. Vibration analysis of submerged rectangular microplates with distributed mass loading. Proc. Royal Society, Series B, 465: 1323-1336. DOI: 10.1098/rspa..0447

Young, D., 1950. Vibration of rectangular plates by the Ritz method. J. Applied Mechan., 17: 448-453.

Zhong, Y., X.F. Zhao and R. Li, 2013. Free vibration analysis of rectangular cantilever plates by finite integral transform method. Int. J. Comput. Methods Eng. Sci. Mechan., 14: 221-226. DOI: $10.1080 / 15502287.2012 .711424$ 